\title{
Biogeography and conservation of the herpetofauna of the Upland Pine-Oak Forests of Honduras
}

\author{
Larry David Wilson ${ }^{1,3}$ \& Josiah Harold Townsend ${ }^{2}$ \\ Biota Neotropica $v 7$ (n1) - http://www.biotaneotropica.org.br/v7n1/pt/abstract?inventory+bn02307012007 \\ Data Received 09/06/06 \\ Revised 01/12/06 \\ Accepted 20/01/07 \\ ${ }^{1}$ Department of Biology, Kendall Campus, Miami-Dade College, Miami, Florida, 33176-3393, USA \\ ${ }^{2}$ School of Natural Resources and Environment, University of Florida, and Division of Herpetology, \\ Florida, Museum of Natural History, Gainesville, Florida, 32611-7800, USA, \\ e-mail: jtownsend@flmnh.ufl.edu \\ ${ }^{3}$ Corresponding author: Larry David Wilson,e-mail: lwilson@mdc.edu
}

\begin{abstract}
Wilson, L.D. \& Townsend, J.H. Biogeography and conservation of the herpetofauna of the Upland Pine-Oak Forests of Honduras. Biota Neotrop. Jan/Apr 2007 vol. 7, no. 1 http://www.biotaneotropica.org. br/v7n1/pt/abstract?inventory+bn02307012007 ISSN 1676-0603.

The upland pine-oak forest herpetofauna constitutes the smallest segment distributed in the major habitat types in Honduras, due to its occurrence at moderate elevations in relatively inhospitable environments, compared to more mesic habitats in the country. This segment, however, is subject to considerable environmental threat as a consequence of annual burning and logging. Of the 356 herpetofaunal species known from Honduras, 105 are known from these habitats. These forests occur throughout much of the mountainous interior of Honduras. They are subject to the Intermediate Dry climate. Four salamanders, 27 anurans, four turtles, 29 lizards, and 41 snakes comprise the herpetofauna. These species are partitioned into restricted, widespread, and peripheral distributional categories. They can be allocated to eleven broad distributional categories, with most belonging to the category containing species whose ranges extend from somewhere in Mexico north of the Isthmus of Tehuantepec to somewhere in South America. The large majority are terrestrial forest inhabitants, arboreal forest inhabitants, terrestrial pondside species, or terrestrial forest pondside species. Most species are judged common, with the next largest group considered to be of infrequent occurrence, and the smallest group of rare occurrence. Upland pine-oak forest species are distributed among four ecophysiographic areas, with the greatest number of species being found in the Southeastern Uplands. Construction of a CBR diagram illustrates that the herpetofaunas of the Northwestern and Northeastern Uplands, the Northeastern Uplands and Southeastern Uplands, and the Southeastern Uplands and Southwestern Uplands are about equally related to one another. The greatest significance of the upland pineoak forest herpetofauna lies in the relatively high percentage of members presently possessing stable populations, indicating their apparent greater ability to resist anthropogenic habitat disturbance. Few species currently have populations in decline, but about a third lack sufficient data to characterize their population status, indicating the need for considerable additional fieldwork before their conservation issues can be properly addressed.
\end{abstract}

Keywords: Amphibians and reptiles, distributional patterns, biodiversity significance, conservation status.

\section{Resumen}

Wilson, L.D. \& Townsend, J.H. “Biodiversidad y conservación de la herpetofauna de los Bosques de PinoRoble de la Altiplanicie de Honduras. Biota Neotrop. Jan/Apr 2007 vol. 7, no. 1 http://www.biotaneotropica. org.br/v7n1/pt/abstract?inventory+bn02307012007 ISSN 1676-0603.

La herpetofauna de los bosques de pino-roble del altiplano constituye el segmento más pequeño distribuido en los habitats principales de Honduras, debido a su ocurrencia a elevaciones moderadas en ambientes relativamente inhóspitos, especialmente comparados a los ambientes más húmedos de país. Este segmento, sin embargo, está sujeto a una amenaza ambiental considerable, debido a las quemas anuales y a la tala de árboles. De las 356 especies de herpetofauna conocidas en Honduras, 105 son de estos habitats. Esta clase de bosques es prevalente en gran parte del interior montañoso de Honduras. Están sujetos al clima Seco Intermedio. La herpetofauna consiste de 4 salamandras, 27 anuros, cuatro tortugas, 29 lagartijas, y 41 serpientes. Estas especies se clasifican en categorías de distribución restrictas, extensas, y periféricas. Es posible colocar estas especies en once categorías de distribución, con la mayoría encontrándose en la categoría que contiene las especies cuyos rangos se extienden desde alguna parte de México al norte del Istmo de Tehuantepec hasta alguna parte de Suramérica. La gran mayoría son habitantes terrestres del bosque, habitantes arbóreos del bosque, especies terrestres de la orilla de estanques de agua, o especies terrestres del bosque encontradas a la orilla de estanques de agua. La mayoría de las especies se consideran comunes, con el próximo grupo más grande considerado como de ocurrencia poco frecuente, y el 
grupo más pequeño de rara ocurrencia. Las especies de bosques de pino y roble del altiplano están distribuidas en cuatro áreas ecofisiográficas, con el mayor número de especies encontradas en los altiplanos del sureste. La creación de un diagrama de Coeficientes de Semejanza Biogeográfica (CBR por sus siglas en inglés) ilustra que la herpetofauna de los altiplanos del noroeste y del nordeste, la de los altiplanos del nordeste y del sureste, y la de los altiplanos del sureste y del suroeste, están relacionadas entre sí en forma equitativa. Lo más importante sobre la herpetofauna del bosque de pino-roble del altiplano yace en su porcentaje relativamente alto de los miembros que actualmente poseen poblaciones estables, indicando, aparentemente, una mayor capacidad para resistir disturbios antropogénicos del ambiente. Actualmente hay pocas especies que tienen poblaciones en declive, pero alrededor de un tercio carecen de suficiente información para caracterizar su estado de población, indicando la necesidad del trabajo del campo adicional significativo antes de poder tratar los asuntos de su conservación correctamente.

Palabras-clave: anfibios y reptiles, patrones de distribución, importancia en la biodiversidad, estado de conservación.

\section{Introduction}

Forests of varying combinations of pine and oak species occur broadly at moderate elevations $(600-1500 \mathrm{~m})$ throughout Mexico and northern Central America (Wagner 1964, Farjon et al., 1997). In Honduras, forests of Pinus oocarpa and Quercus spp. are widely distributed through the mountainous interior in upland environments (McCranie \& Wilson 2002). The climate in the areas supporting pine-oak forest is typically cooler and drier than that found in the lowlands, and, therefore, human inhabitants have favored these areas since pre-Colombian times. Consequently, these forests have probably seen the most consistent and long-term degradation at the hands of humans of any forested area of Honduras.

These forests are inhabited by a relatively small herpetofauna, compared to that of rainforests (Wilson \& Townsend 2006). The members of the upland pine-oak forest herpetofauna generally possess relatively broad geographic distributions both within Honduras and outside the country. As a consequence, there is a relatively small amount of endemism associated with upland pine-oak forests, with only one species of snake (Tantilla lempira) essentially limited to this habitat type. Nevertheless, these forests and their constituent herpetofaunas are of considerable interest, allowing for study of what species of Honduran amphibians and reptiles are able to persist in an environment that is relatively dry and inhospitable to at least amphibians and subject to virtually annual burning, either by natural or anthropogenic means, and to widespread logging.

\section{Materials and Methods}

Fieldwork on which this paper is based has been conducted by one of us (LDW) since 1967 and the other (JHT) since 1999 (also see Acknowledgments), in collaboration with James R. McCranie and other researchers (see Acknowledgments). This fieldwork was primarily conducted during the summer months (May through August). The material collected has been reported in a number of publications by the first author and coauthors since 1971, including Meyer \& Wilson (1971, 1973), Wilson \& Meyer (1985), McCranie \& Wilson (2002), and McCranie et al. (2006).

In constructing this paper, we have followed the format used by Wilson \& McCranie (2004a) in their study of the herpetofauna of the cloud forests of Honduras, and Wilson \& Townsend (2006, In press), in their studies of the herpetofauna of the rainforests and subhumid forests of Honduras.

As did Wilson \& Meyer (1985), Wilson et al. (2001), McCranie \& Wilson (2002), Wilson \& McCranie (2004a), and Wilson \& Townsend (2006, In press), we allocated the upland pine-oak species to a set of distributional categories based on the entire extent of their geographic range. In addition to the categories used by Wilson et al. (2001), slightly modified by us, we have added another category containing the species introduced to the herpetofauna of Honduras. The categories are as follows: $\mathrm{A}=$ Northern terminus of the range in the United States (or Canada) and southern terminus in South America; B = Northern terminus of the range in the United States and southern terminus in Central America south of the Nicaraguan Depression; $\mathrm{C}=$ Northern terminus of the range in the United States and southern terminus in Nuclear Middle America; $\mathrm{D}=$ Northern terminus of the range in Mexico north of the Isthmus of Tehuantepec and southern terminus in South America; $\mathrm{E}=$ Northern terminus of the range in Mexico north of the Isthmus of Tehuantepec and southern terminus in Central America south of the Nicaraguan Depression; F = Northern terminus of the range in Mexico north of the Isthmus of Tehuantepec and southern terminus in Nuclear Middle America; $G=$ Northern terminus of the range in Nuclear Middle America and southern terminus in South America; $\mathrm{H}=$ Northern terminus of the range in Nuclear Middle America and southern terminus in Central America south of the Nicaraguan Depression; I = Restricted to Nuclear Middle America (exclusive of Honduran endemics); $\mathrm{J}=$ Endemic to Honduras; $\mathrm{K}=$ Marine species (not included in this paper); $\mathrm{L}=$ Insular species (includes non-endemic species limited to insular habitats in Honduras; not included in this paper); $\mathrm{M}=$ Introduced species.

When characterizing the primary microhabitat distribution of each species, we have used the same microhabitat categorization as did Wilson \& McCranie (2004a) and Wilson \& Townsend (2006, In press). With respect to vertical positioning, we scored species as either terrestrial or arboreal. With regard to occurrence in the three major microhabitats found in upland pine-oak forests, species were scored as being found in the forest proper, along streams, or around ponds.

In examining relative abundance, we used the following categories: common (found on a regular basis, many individuals can be found); infrequent (unpredictable, few individuals seen); rare (rarely seen). These classifications are historical (i.e., based on earlier trips to upland pine-oak forest localities) and do not take into consideration the more recent population declines taking place for a number of species.

Conservation status categorization was determined on the basis of observations made during recent fieldwork. As such, these determinations are admittedly provisional.

The Coefficient of Biogeographic Resemblance algorithm (Duellman 1990) was used to demonstrate herpetofaunal relationships among the upland pine-oak forest ecophysiographic areas examined in this study. The formula is $\mathrm{CBR}=2 \mathrm{C} /\left(\mathrm{N}_{1}+\mathrm{N}_{2}\right)$, where $\mathrm{C}$ is the number of species in common to both formations, $\mathrm{N}_{1}$ is the number of species in the first area, and $\mathrm{N}_{2}$ is the number of species in the second area. 
There are some recent taxonomic changes that we use in this paper that differ from previous papers in this series (Wilson \& McCranie 2004a, Wilson \& Townsend 2006). At the generic level, the beta anoles, previously referred to as Norops (sensu Guyer \& Savage $(1986,1992))$, are placed within the super-genus Anolis following Poe (2004) and in anticipation of further taxonomic revision. Chaunus and Ollotis are used in place of Bufo (Frost et al. 2006a, b), Craugastor is used instead of Eleutherodactylus for Central American species (Crawford \& Smith 2005), Dendropsophus, Tlalocohyla, and Trachycephalus replace Hyla (Faivovich et al. 2005), Lithobates replaces Rana for Central American species (Frost et al. 2006), and Mastigodryas is used in favor of Dryadophis (Dixon \& Tipton 2004). At the species level, Lithobates brownorum is used for Honduran populations formerly referred to as Lithobates or Rana berlandieri (Zaldívar-Riverón et al. 2004, Frost 2004, McCranie et al. 2006), and Atropoides mexicanus replaces A. nummifer (Campbell \& Lamar 2004).

\section{Description of Study Area}

\section{Physiography}

The largest portion of Honduras, the mountainous interior, is a component of Nuclear Middle America, the complex of mountains distributed south of the Mexican Isthmus of Tehuantepec to north of the Nicaraguan Depression. The Honduran segment of this mountain complex belongs to what Campbell (1999) termed "eastern Nuclear Central America." The moderate elevations (about 600 to about $1500 \mathrm{~m}$ ) of these Honduran mountains support what we term upland pine-oak forest (see discussion under "Vegetation").

In Honduras, the mountainous interior is termed the "serranía," and can be divided into a northern portion, the Northern Cordillera, and a southern portion, the Southern Cordillera, distinguished from one another on the basis of the presence of Pliocene volcanic ejecta in the latter region (McCranie \& Wilson 2002). Both of these cordilleras are divided into western and eastern portions by the extent of the Honduran Depression, a north-south complex of plains and valleys, including the Ulúa-Chamelecón Plain, the Humuya Valley, the Comayagua Plain, and the Goascorán Valley (McCranie \& Wilson 2002). These physiographic divisions allow for the recognition of four upland areas that support pine-oak forest, viz., the Northwestern, Southwestern, Northeastern, and Southeastern Uplands (Figure 1).

\section{Climate}

One major type of climate characterizes the upland pine-oak forests of Honduras. This type is referred to as the Intermediate Dry climate and occurs at elevations from about 600 to $1500 \mathrm{~m}$ and, in some locales, to about $1850 \mathrm{~m}$ (McCranie \& Wilson 2002). It features a mean annual precipitation of 1000 to $2000 \mathrm{~mm}$ and a mean annual temperature ranging from 18 to $24{ }^{\circ} \mathrm{C}$.

\section{Vegetation}

Upland pine-oak forest in Honduras is allocated to the Premontane Moist Forest formation of Holdridge (1967). As suggested by its name, this type of forest is dominated by species of pines and oaks. The principal pine species in question is Pinus oocarpa, a species distributed from northwestern Mexico to northwestern Nicaragua (Farjon et al. 1997). Other species of pines also occur in the upland pine-oak forests of Honduras, including $P$. maximinoi, P. pseudostrobus, and P. tecunumanii (Farjon et al. 1997). The two common species of oaks are Quercus oleoides, a small-leaf species characteristic of encinales, and $Q$. peduncularis, a large-leaf species found in robledales (Agüdelo C. 1987). Additional tree spe-

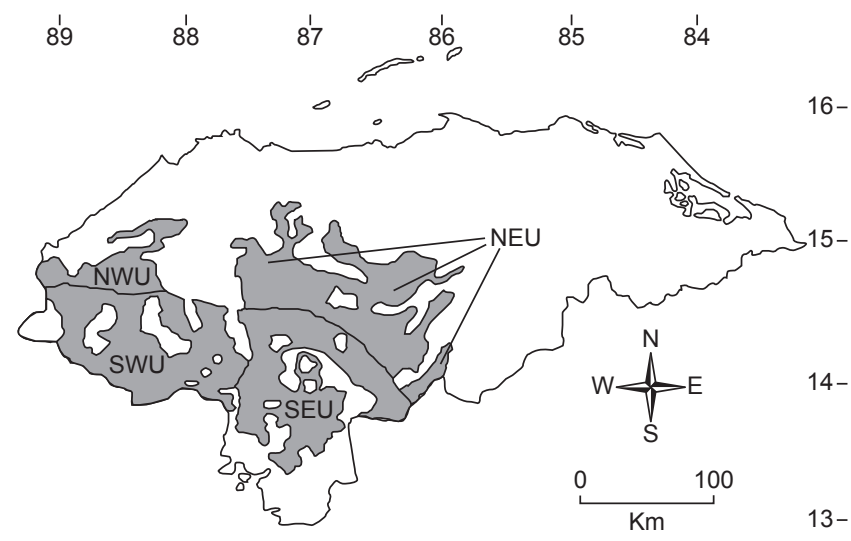

Figure 1. Generalized map of the upland pine-oak forest areas of Honduras. Abbreviations are as follows: $\mathrm{NWU}=$ Northwestern Uplands; $\mathrm{NEU}=$ Northeastern Uplands; SWU $=$ Southwestern Uplands; SEU $=$ Southeastern Uplands.

Figura 1. Mapa general de las áreas de bosques de pino-roble de altiplano en Honduras. Las abreviaturas son las siguientes, de acuerdo sus siglas en inglés: $\mathrm{NWU}=$ Altiplano del Noroeste; $\mathrm{NEU}=$ Altiplano del Nordeste; SWU = Altiplano del Suroeste; SEU = Altiplano del Sureste .

cies present in upland pine-oak forests include Arbutus xalapensis, Brysonima crassifolia, Curatella americana, Dodonaea viscosa, Genipa caruto, Lysiloma seemannii, Paurotis cookii, and Piscidia grandifolia (Agüdelo C. 1987; McCranie \& Wilson 2002).

\section{Results}

\section{Composition of the upland pine-oak forest herpetofauna}

The herpetofauna of the upland pine-oak forests of Honduras currently is known to comprise 105 species (Table 1), including four salamanders (3.8\% of total), 27 anurans $(25.7 \%)$, four turtles (3.8\%), 29 lizards (27.6\%), and 41 snakes (39.1\%). The salamanders all belong to the family Plethodontidae. The anurans belong to six families, including the Brachycephalidae (three species), Bufonidae (five species), Centrolenidae (one species), Hylidae (eight species), Leptodactylidae (four species), Microhylidae (two species), and Ranidae (four species). The turtles are members of three families, including the Emydidae (one species), Geoemydidae (one species), and Kinosternidae (two species). The lizards are members of nine families, including the Anguidae (two species), Gekkonidae (four species), Corytophanidae (three species), Iguanidae (two species), Phrynosomatidae (three species), Polychrotidae (eight species), Scincidae (three species), Gymnophthalmidae (one species), and Teiidae (three species). The snakes belong to seven families, including the Leptotyphlopidae (one species), Typhlopidae (one species), Boidae (one species), Ungaliophiidae (one species), Colubridae (34 species), Elapidae (one species), and Viperidae (two species).

\section{Distribution and distributional relationships of the upland pine-oak forest herpetofauna}

1. Distribution within the upland pine-oak forest. The species occupying the upland pine-oak forests are distributed among three distributional categories, i.e., restricted, widespread, and peripheral (Table 1). Restricted species are those whose distribution in Honduras is limited to the upland pine-oak forest. Widespread species are those distributed within this formation, as well as in other forest formations. Finally, peripheral species are those whose distribution lies largely outside of the upland pine-oak forest. 
Table 1. Geographic and ecological distribution, relative abundance, and conservation status of the upland pine-oak forest herpetofauna (105 species) of Honduras. Abbreviations include: Forest Formation Distribution-W = widespread in that formation, $\mathrm{R}=$ restricted to that formation, $\mathrm{P}=$ peripherally distributed in that formation; Broad Distributional Patterns-A = Northern terminus of the range in the United States (or Canada) and southern terminus in South America; $\mathrm{B}=$ Northern terminus of the range in the United States and southern terminus in Central America south of the Nicaraguan Depression; $\mathrm{C}=$ Northern terminus of the range in the United States and southern terminus in Nuclear Middle America; D = Northern terminus of the range in Mexico north of the Isthmus of Tehuantepec and southern terminus in South America; $\mathrm{E}=$ Northern terminus of the range in Mexico north of the Isthmus of Tehuantepec and southern terminus in Central America south of the Nicaraguan Depression; F = Northern terminus of the range in Mexico north of the Isthmus of Tehuantepec and southern terminus in Nuclear Middle America; $\mathrm{G}=$ Northern terminus of the range in Nuclear Middle America and southern terminus in South America; $\mathrm{H}=\mathrm{Northern}$ terminus of the range in Nuclear Middle America and southern terminus in Central America south of the Nicaraguan Depression; I = Restricted to Nuclear Middle America (exclusive of Honduran endemics); $\mathrm{J}=$ Endemic to Honduras; $\mathrm{M}=$ Introduced species; Primary Microhabitat $-\mathrm{Ar}=\operatorname{arboreal}, \mathrm{Te}=$ terrestrial, Fo $=$ forest inhabitant, $\mathrm{Ps}=$ pondside inhabitant, $\mathrm{St}=$ streamside inhabitant; Relative Abundance - Common $=$ found on a regular basis, many individuals can be found; Infrequent = encountered unpredictably, few individuals seen; Rare = rarely seen; Conservation Status - Stable = stable populations at least at one upland pine-oak forest locality, Declining = all known upland pine-oak forest populations declining, Extinct $=$ extinct or extirpated from all known upland pine-oak forest localities, No Data = insufficient data available on population status.

Tabla 1. Distribución geográfica y ecológica, abundancia relativa, y estado de conservación de la herpetofauna de los bosques de pino-roble de altiplano de Honduras (105 especies). Las abreviaturas incluyen, de acuerdo sus siglas en inglés: Distribución de Zonas de Vida Boscosas-W = distribución extensa en esa zona de vida, $\mathrm{R}=$ limitado a esa zona de vida, $\mathrm{P}=$ distribución periférica en esa zona de vida; Patrones Amplios de Distribución-A = Terminal norte de extensión en los Estados Unidos (o Canadá), y terminal sur en Sur América; B = Terminal norte de extensión en los Estados Unidos, y terminal sur en Centro América, al sur de la Depresión Nicaragüense; $\mathrm{C}=$ Terminal norte de extensión en los Estados Unidos, y terminal sur en el Núcleo de Centroamérica; D = Terminal norte de extensión en México, al norte del Istmo de Tehuantepec, y terminal sur en Sur América; E = Terminal norte de extensión en México al norte del Istmo de Tehuantepec, y terminal sur en Centro América, al sur de la Depresión Nicaragüense; $\mathrm{F}=$ Terminal norte de extensión en México al norte del Istmo de Tehuantepec, y terminal sur en el Núcleo de Centroamérica; $\mathrm{G}=$ Terminal norte de extensión en el Núcleo de Centroamérica, y terminal sur en Sur América; $\mathrm{H}$ = Terminal norte de extensión en el Núcleo de Centroamérica, y terminal sur en Centro América, al sur de la Depresión Nicaragüense; I = Limitado al Núcleo de Centroamérica (excluyendo las endémicas hondureñas); $\mathrm{J}=$ Endémica a Honduras; $\mathrm{M}=$ Especie introducida; Microhábitat Primario- $\mathrm{Ar}=$ arborícola, $\mathrm{Te}=$ terrestre, $\mathrm{Fo}=$ habitante del bosque, $\mathrm{Ps}=$ habitante de orilla de estanque, $\mathrm{St}=$ habitante de orilla de arroyo; Abundancia Relativa - Común = se encuentra con regularidad, se encuentran muchos individuos; Poco frecuente = no se puede predecir cuando se encontrará, se ven pocos ejemplares; Raro = rara vez se ve; Estado de Conservación-Estable = Poblaciones estables en por lo menos una localidad con bosques de pino-roble de altiplano, Descenso $=$ todas las poblaciones conocidas en el bosque de pino-roble de altiplano están en descenso, Extinto = Extinto o extirpado de todas las localidades de bosque de pino-roble de altiplano reconocidas, No Data = no hay suficiente data disponible sobre el estado de la población.

\begin{tabular}{|c|c|c|c|c|c|c|}
\hline Species & $\begin{array}{c}\text { Forest } \\
\text { formation } \\
\text { distribution }\end{array}$ & $\begin{array}{l}\text { Elevational } \\
\text { range }(\mathrm{m})\end{array}$ & $\begin{array}{c}\text { Broad } \\
\text { distribution } \\
\text { pattern } \\
\end{array}$ & $\begin{array}{c}\text { Primary } \\
\text { microhabitat }\end{array}$ & $\begin{array}{c}\text { Relative } \\
\text { abundance }^{1}\end{array}$ & $\begin{array}{c}\text { Conservation } \\
\text { status }\end{array}$ \\
\hline \multicolumn{7}{|c|}{ Salamanders (4 species) } \\
\hline Bolitoglossa mexicana & $\mathrm{P}$ & $0-1400$ & $\mathrm{~F}$ & Ar, Fo & Common & Stable \\
\hline Bolitoglossa occidentalis & $\mathrm{R}$ & 1300 & $\mathrm{I}$ & Ar, Fo & Rare & No Data \\
\hline Bolitoglossa rufescens & $\mathrm{W}$ & $30-1400$ & I & Ar, Fo & Common & Stable \\
\hline Oedipina ignea & $\mathrm{W}$ & $1340-1750$ & I & Te, Fo & Infrequent & Stable \\
\hline \multicolumn{7}{|c|}{ Anurans (27 species) } \\
\hline Chaunus marinus & $\mathrm{W}$ & $0-1435$ & $\mathrm{~A}$ & Te, Fo, Ps & Common & Stable \\
\hline Ollotis coccifer & $\mathrm{W}$ & $0-1435$ & $\mathrm{E}$ & Te, Ps & Common & Stable \\
\hline Ollotis ibarrai & $\mathrm{W}$ & $1280-2020$ & $\mathrm{E}$ & Te, Ps & Common & Stable \\
\hline Ollotis luetkenii & $\mathrm{W}$ & $0-1100$ & $\mathrm{H}$ & Te, Ps & Common & Stable \\
\hline Ollotis valliceps & $\mathrm{W}$ & $0-1610$ & $\mathrm{E}$ & Te, Fo, Ps & Common & Stable \\
\hline Hyalinobatrachium fleischmanni & $\mathrm{W}$ & $0-1550$ & $\mathrm{D}$ & $\mathrm{Ar}, \mathrm{St}$ & Common & No Data \\
\hline Dendropsophus microcephalus & $\mathrm{W}$ & $0-1000$ & $\mathrm{~B}$ & Ar, Ps & Common & Stable \\
\hline Plectrohyla guatemalensis & $\mathrm{P}$ & $950-2600$ & I & $\mathrm{Ar}, \mathrm{St}$ & Infrequent & Declining \\
\hline Ptychohyla hypomykter & $\mathrm{W}$ & $620-2070$ & I & $\mathrm{Ar}, \mathrm{St}$ & Common & Stable \\
\hline Ptychohyla salvadorensis & $\mathrm{P}$ & $1440-2050$ & I & $\mathrm{Ar}, \mathrm{St}$ & Common & Stable \\
\hline Scinax staufferi & $\mathrm{W}$ & $0-1530$ & $\mathrm{E}$ & Ar, Te, Ps & Common & Stable \\
\hline Smilisca baudinii & W & $0-1610$ & $\mathrm{~B}$ & Ar, Ps & Common & Stable \\
\hline Tlalocohyla loquax & W & $0-1585$ & $\mathrm{E}$ & Ar, Ps & Common & Stable \\
\hline Trachycephalus venulosus & W & $0-1610$ & $\mathrm{D}$ & Ar, Te, Ps & Common & Stable \\
\hline Craugastor anciano & $\mathrm{P}$ & $1400-1840$ & $\mathrm{~J}$ & $\mathrm{Te}, \mathrm{St}$ & Rare & Extinct \\
\hline Craugastor emleni & $\mathrm{W}$ & $800-2000$ & $\mathrm{~J}$ & $\mathrm{Te}, \mathrm{St}$ & Common & Extinct \\
\hline Craugastor laevissimus & W & $100-1640$ & $\mathrm{H}$ & $\mathrm{Te}, \mathrm{St}$ & Common & Stable \\
\hline Leptodactylus fragilis & W & $0-1530$ & A & Te, Ps & Common & Stable \\
\hline Leptodactylus melanonotus & W & $0-1280$ & $\mathrm{D}$ & Te, Ps & Common & Stable \\
\hline Leptodactylus silvanimbus & W & $1470-2000$ & $\mathrm{~J}$ & Te, Ps & Infrequent & Declining \\
\hline Engystomops pustulosus & $\mathrm{W}$ & $0-1540$ & $\mathrm{D}$ & Te, Ps & Common & Stable \\
\hline
\end{tabular}


Table 1. Continued....

\begin{tabular}{|c|c|c|c|c|c|c|}
\hline Species & $\begin{array}{c}\text { Forest } \\
\text { formation } \\
\text { distribution }\end{array}$ & $\begin{array}{c}\text { Elevational } \\
\text { range }(\mathbf{m})\end{array}$ & $\begin{array}{c}\text { Broad } \\
\text { distribution } \\
\text { pattern } \\
\end{array}$ & $\begin{array}{c}\text { Primary } \\
\text { microhabitat }\end{array}$ & $\begin{array}{c}\text { Relative } \\
\text { abundance }^{1}\end{array}$ & $\begin{array}{c}\text { Conservation } \\
\text { status }\end{array}$ \\
\hline Hypopachus barberi & $\mathrm{W}$ & $1470-2070$ & $\mathrm{I}$ & Te, Ps & Infrequent & No Data \\
\hline Hypopachus variolosus & W & $0-1610$ & $\mathrm{~B}$ & Te, Ps & Common & Stable \\
\hline Lithobates brownorum & $\mathrm{W}$ & $0-1650$ & $\mathrm{C}$ & Te, Ps & Common & Stable \\
\hline Lithobates forreri & $\mathrm{P}$ & $35-1000$ & $\mathrm{E}$ & $\mathrm{Te}, \mathrm{Ps}$ & Common & Stable \\
\hline Lithobates maculatus & $\mathrm{W}$ & $40-1980$ & I & $\mathrm{Te}, \mathrm{St}$ & Common & No Data \\
\hline Lithobates vaillanti & $\mathrm{P}$ & $0-880$ & $\mathrm{D}$ & $\mathrm{Te}, \mathrm{Ps}$ & Common & Stable \\
\hline \multicolumn{7}{|c|}{ Turtles (4 species) } \\
\hline Trachemys venusta & $\mathrm{P}$ & $0-650$ & $\mathrm{D}$ & $\mathrm{Te}, \mathrm{Fo}, \mathrm{Ps}, \mathrm{St}$ & Infrequent & No Data \\
\hline Rhinoclemmys pulcherrima & $\mathrm{P}$ & $0-1480$ & $\mathrm{E}$ & Te, Fo & Infrequent & Stable \\
\hline Kinosternon leucostomum & $\mathrm{W}$ & $0-1120$ & $\mathrm{D}$ & Te, Fo, Ps & Infrequent & No Data \\
\hline Kinosternon scorpioides & $\mathrm{W}$ & $0-1240$ & $\mathrm{D}$ & Te, Fo, Ps & Common & Stable \\
\hline \multicolumn{7}{|c|}{ Lizards (29 species) } \\
\hline Celestus bivittatus & $\mathrm{W}$ & $1510-1980$ & I & Te, Fo & Common & Stable \\
\hline Celestus scansorius & $\mathrm{P}$ & $1550-1590$ & $\mathrm{~J}$ & Ar, Fo & Rare & No Data \\
\hline Hemidactylus frenatus & W & $0-930$ & M & $\mathrm{Ar}, \mathrm{Fo}$ & Common & Stable \\
\hline Phyllodactylus tuberculosus & $\mathrm{W}$ & $0-1200$ & $\mathrm{E}$ & Ar, Fo & Common & Stable \\
\hline Sphaerodactylus glaucus & $\mathrm{R}$ & 600 & $\mathrm{~F}$ & $\mathrm{Te}, \mathrm{Fo}$ & Rare & No Data \\
\hline Sphaerodactylus millepunctatus & $\mathrm{W}$ & $0-930$ & $\mathrm{H}$ & Te, Fo & Common & Stable \\
\hline Basiliscus vittatus & $\mathrm{W}$ & $0-1400$ & $\mathrm{D}$ & $\mathrm{Ar}, \mathrm{Fo}, \mathrm{St}$ & Common & Stable \\
\hline Corytophanes percarinatus & $\mathrm{R}$ & 1400 & I & Te, Fo & Rare & No Data \\
\hline Laemanctus longipes & $\mathrm{P}$ & $0-1200$ & $\mathrm{~F}$ & Ar, Fo & Infrequent & No Data \\
\hline Ctenosaura similis & $\mathrm{P}$ & $0-1200$ & $\mathrm{H}$ & Ar, Fo & Common & No Data \\
\hline Iguana iguana & W & $0-880$ & $\mathrm{D}$ & $\mathrm{Ar}, \mathrm{Fo}, \mathrm{St}$ & Common & No Data \\
\hline Sceloporus malachiticus & $\mathrm{W}$ & $540-2530$ & $\mathrm{H}$ & Ar, Fo & Common & Stable \\
\hline Sceloporus olloporus & W & $0-1510$ & $\mathrm{~B}$ & Ar, Fo & Common & Stable \\
\hline Sceloporus squamosus & $\mathrm{W}$ & $0-1000$ & $\mathrm{H}$ & $\mathrm{Ar}, \mathrm{Te}, \mathrm{Fo}$ & Common & Stable \\
\hline Anolis biporcatus & $\mathrm{P}$ & $0-950$ & $\mathrm{D}$ & Ar, Fo & Infrequent & No Data \\
\hline Anolis crassulus & $\mathrm{W}$ & $1200-2020$ & I & Ar, Fo & Common & Stable \\
\hline Anolis dariense & $\mathrm{W}$ & $0-1250$ & I & $\mathrm{Ar}, \mathrm{Te}, \mathrm{Fo}$ & Common & Stable \\
\hline Anolis laeviventris & $\mathrm{W}$ & $1000-1900$ & $\mathrm{E}$ & Ar, Fo & Common & Stable \\
\hline Anolis rodriguezii & $\mathrm{P}$ & $0-1050$ & $\mathrm{~F}$ & $\mathrm{Ar}, \mathrm{Te}, \mathrm{Fo}, \mathrm{St}$ & Infrequent & Stable \\
\hline Anolis sericeus & W & $0-1320$ & $\mathrm{E}$ & Ar, Fo & Common & Stable \\
\hline Anolis sminthus & $\mathrm{P}$ & $1450-2200$ & $\mathrm{~J}$ & Ar, Fo & Infrequent & Stable \\
\hline Anolis tropidonotus & $\mathrm{W}$ & $0-1900$ & $\mathrm{~F}$ & Ar, Te, Fo & Common & Stable \\
\hline Mabuya unimarginata & W & $0-1510$ & $\mathrm{D}$ & Ar, Te, Fo & Common & Stable \\
\hline Mesoscincus managuae & W & $0-920$ & $\mathrm{H}$ & Te, Fo & Rare & No Data \\
\hline Sphenomorphus cherriei & W & $0-1860$ & $\mathrm{E}$ & Te, Fo & Common & Stable \\
\hline Gymnophthalmus speciosus & W & $0-1320$ & G & Te, Fo & Infrequent & No Data \\
\hline Ameiva undulata & W & $0-1240$ & $\mathrm{E}$ & Te, Fo & Common & Stable \\
\hline Aspidoscelis deppii & W & $0-900$ & $\mathrm{E}$ & Te, Fo & Common & Stable \\
\hline Aspidoscelis motaguae & $\mathrm{W}$ & $50-950$ & $\mathrm{~F}$ & Te, Fo & Common & Stable \\
\hline \multicolumn{7}{|c|}{ Snakes (41 species) } \\
\hline Leptotyphlops phenops & $\mathrm{P}$ & $0-700$ & $\mathrm{D}$ & Te, Fo & Infrequent & No Data \\
\hline Typhlops costaricensis & W & $150-1500$ & $\mathrm{H}$ & Te, Fo & Rare & No Data \\
\hline Boa constrictor & $\mathrm{W}$ & $0-1370$ & $\mathrm{D}$ & Te, Fo & Common & Stable \\
\hline Ungaliophis continentalis & W & $990-1600$ & I & Ar, Fo & Rare & No Data \\
\hline Adelphicos quadrivirgatum & $\mathrm{W}$ & $0-1740$ & $\mathrm{~F}$ & Te, Fo & Common & Stable \\
\hline Coniophanes fissidens & $\mathrm{W}$ & $0-1300$ & $\mathrm{D}$ & Te, Fo & Common & Stable \\
\hline
\end{tabular}


Table 1. Continued....

\begin{tabular}{|c|c|c|c|c|c|c|}
\hline Species & $\begin{array}{c}\text { Forest } \\
\text { formation } \\
\text { distribution }\end{array}$ & $\begin{array}{c}\text { Elevational } \\
\text { range }(\mathbf{m})\end{array}$ & $\begin{array}{c}\text { Broad } \\
\text { distribution } \\
\text { pattern }\end{array}$ & $\begin{array}{c}\text { Primary } \\
\text { microhabitat }\end{array}$ & $\begin{array}{c}\text { Relative } \\
\text { abundance }\end{array}$ & $\begin{array}{c}\text { Conservation } \\
\text { status }\end{array}$ \\
\hline Coniophanes piceivittis & $\mathrm{W}$ & $30-1280$ & $\mathrm{E}$ & $\mathrm{Te}, \mathrm{Fo}$ & Infrequent & No Data \\
\hline Conophis concolor & $\mathrm{W}$ & $0-1000$ & $\mathrm{E}$ & Te, Fo & Common & Stable \\
\hline Dendrophidion percarinatum & $\mathrm{P}$ & $20-685$ & G & $\mathrm{Te}, \mathrm{Fo}$ & Infrequent & No Data \\
\hline Drymarchon melanurus & $\mathrm{W}$ & $0-1555$ & A & $\mathrm{Te}, \mathrm{Fo}$ & Common & Stable \\
\hline Drymobius margaritiferus & $\mathrm{W}$ & $0-1450$ & A & Te, Fo, Ps & Common & Stable \\
\hline Enulius flavitorques & $\mathrm{W}$ & $0-1000$ & $\mathrm{D}$ & Te, Fo & Common & Stable \\
\hline Imantodes cenchoa & $\mathrm{W}$ & $0-1620$ & $\mathrm{D}$ & $\mathrm{Ar}, \mathrm{Fo}$ & Common & Stable \\
\hline Lampropeltis triangulum & $\mathrm{W}$ & $0-1370$ & A & Te, Fo & Infrequent & Stable \\
\hline Leptodeira annulata & $\mathrm{W}$ & $0-1530$ & $\mathrm{D}$ & Te, Fo & Common & Stable \\
\hline Leptodrymus pulcherrimus & $\mathrm{W}$ & $10-1300$ & $\mathrm{H}$ & Te, Fo & Infrequent & No Data \\
\hline Leptophis ahaetulla & $\mathrm{P}$ & $0-1680$ & $\mathrm{D}$ & $\mathrm{Ar}, \mathrm{Te}, \mathrm{Ps}, \mathrm{St}$ & Common & Stable \\
\hline Leptophis mexicanus & $\mathrm{W}$ & $0-1600$ & $\mathrm{E}$ & Ar, Te, Fo & Common & Stable \\
\hline Masticophis mentovarius & $\mathrm{W}$ & $40-1320$ & $\mathrm{D}$ & $\mathrm{Te}, \mathrm{Te}$ & Common & Stable \\
\hline Mastigodryas dorsalis & W & $635-1900$ & I & Te, Fo & Common & No Data \\
\hline Ninia sebae & $\mathrm{W}$ & $0-1650$ & $\mathrm{E}$ & $\mathrm{Te}, \mathrm{Fo}$ & Common & Stable \\
\hline Oxybelis aeneus & $\mathrm{W}$ & $0-1500$ & A & $\mathrm{Ar}, \mathrm{Te}, \mathrm{Fo}$ & Common & Stable \\
\hline Pliocercus elapoides & $\mathrm{P}$ & $30-1670$ & $\mathrm{~F}$ & Te, Fo & Infrequent & No Data \\
\hline Rhadinaea godmani & $\mathrm{P}$ & $1450-2160$ & $\mathrm{H}$ & Te, Fo & Infrequent & No Data \\
\hline Rhadinaea kinkelini & $\mathrm{W}$ & $1370-2085$ & I & $\mathrm{Te}, \mathrm{Fo}$ & Infrequent & No Data \\
\hline Scolecophis atrocinctus & $\mathrm{W}$ & $700-750$ & $\mathrm{H}$ & Te, Fo & Infrequent & No Data \\
\hline Senticolis triaspis & $\mathrm{W}$ & $100-1100$ & $\mathrm{~B}$ & Te, Fo & Infrequent & No Data \\
\hline Sibon dimidiatus & $\mathrm{P}$ & $950-1600$ & $\mathrm{E}$ & Ar, Fo & Infrequent & Stable \\
\hline Spilotes pullatus & $\mathrm{W}$ & $0-900$ & $\mathrm{D}$ & Ar, Fo & Common & Stable \\
\hline Stenorrhina degenhardtii & $\mathrm{P}$ & $100-1630$ & $\mathrm{D}$ & Te, Fo & Infrequent & No Data \\
\hline Stenorrhina freminvillii & $\mathrm{W}$ & $60-1450$ & $\mathrm{E}$ & Te, Fo & Infrequent & No Data \\
\hline Storeria dekayi & $\mathrm{W}$ & $635-1900$ & $\mathrm{C}$ & Te, Fo & Infrequent & No Data \\
\hline Tantilla armillata & W & $0-1200$ & $\mathrm{H}$ & Te, Fo & Infrequent & No Data \\
\hline Tantilla lempira & $\mathrm{W}$ & $1450-1730$ & $\mathrm{~J}$ & Te, Fo & Rare & No Data \\
\hline Tantilla taeniata & W & $0-1280$ & I & Te, Fo & Infrequent & No Data \\
\hline Thamnophis proximus & W & $0-1000$ & $\mathrm{~B}$ & Te, Fo, Ps & Infrequent & No Data \\
\hline Tretanorhinus nigroluteus & W & $0-750$ & $\mathrm{D}$ & Te, Fo, Ps & Infrequent & No Data \\
\hline Tropidodipsas fischeri & W & $1340-2150$ & I & Te, Fo & Infrequent & No Data \\
\hline Micrurus nigrocinctus & W & $0-1600$ & G & Te, Fo & Common & No Data \\
\hline Crotalus durissus & $\mathrm{W}$ & $20-1300$ & $\mathrm{D}$ & Te, Fo & Infrequent & Declining \\
\hline Porthidium ophryomegas & $\mathrm{W}$ & $0-1400$ & $\mathrm{H}$ & Te, Fo & Infrequent & Declining \\
\hline
\end{tabular}

${ }^{1}$ Historical. For example, species that were common at one time during our field experience, may now be declining or extinct.

The upland pine-oak forests are occupied by three restricted species ( $2.9 \%$ of the total of 105 species), including one salamander and two lizards. Eighty widespread species $(76.2 \%)$ occur in this formation, including two salamanders, 21 anurans, two turtles, 21 lizards, and 34 snakes. Finally, there are 22 species (20.9\%) occurring peripherally in this formation, including one salamander, six anurans, two turtles, six lizards, and seven snakes.

In common with both cloud forest (Wilson \& McCranie 2004a) and rainforest (Wilson \& Townsend 2006) habitats, the largest segment of the upland pine-oak forest herpetofauna comprises the widespread species. On the other hand, there are very few restricted species in the upland pine-oak forest, compared to the situation in both cloud forest and rainforest habitats. Finally, there are relatively more peripheral species in upland pine-oak forests than in rainforests (Wilson \& Townsend 2006) and relatively fewer than in cloud forests (Wilson \& McCranie 2004a).

2. Distribution with respect to elevation. Upland pine-oak forests in Honduras generally occur between the elevations of 600 and $1500 \mathrm{~m}$, although, in some locales they may occur below or above these limits. The restricted species, as a group, occur from 600 to $1400 \mathrm{~m}$. The mean elevation range for this group of three species is zero, inasmuch as the species involved are each known from single localities. The widespread species, as a group, range from sea level to $2530 \mathrm{~m}$. The mean elevational range for this group of 80 species is $1249.5 \mathrm{~m}$. The peripheral species collectively are found from 
Table 2. Summary of numbers of upland pine-oak forest taxa exhibiting various broad patterns of geographic distribution. A = Northern terminus of the range in the United States (or Canada) and southern terminus in South America; B = Northern terminus of the range in the United States and southern terminus in Central America south of the Nicaraguan Depression; $\mathrm{C}=$ Northern terminus of the range in the United States and southern terminus in Nuclear Middle America; D = Northern terminus of the range in Mexico north of the Isthmus of Tehuantepec and southern terminus in South America; E = Northern terminus of the range in Mexico north of the Isthmus of Tehuantepec and southern terminus in Central America south of the Nicaraguan Depression; F = Northern terminus of the range in Mexico north of the Isthmus of Tehuantepec and southern terminus in Nuclear Middle America; G = Northern terminus of the range in Nuclear Middle America and southern terminus in South America; H = Northern terminus of the range in Nuclear Middle America and southern terminus in Central America south of the Nicaraguan Depression; I = Restricted to Nuclear Middle America (exclusive of Honduran endemics); J = Endemic to Honduras; $\mathrm{M}=$ Introduced species.

Tabla 2. Resumen de cantidad de taxa de bosques de pino-roble de altiplano que exhiben patrones amplios y diversos de distribución geográfica. A = Terminal norte de extensión en los Estados Unidos (o Canadá), y terminal sur en Sur América; B = Terminal norte de extensión en los Estados Unidos, y terminal sur en Centro América, al sur de la Depresión Nicaragüense; $\mathrm{C}=$ Terminal norte de extensión en los Estados Unidos, y terminal sur en el Núcleo de Centroamérica; $\mathrm{D}=$ Terminal norte de extensión en México al norte del Istmo de Tehuantepec, y terminal sur en Sur América; E = Terminal norte de extensión en México al norte del Istmo de Tehuantepec, y terminal sur en Centro América, al sur de la Depresión Nicaragüense; F = Terminal norte de extensión en México al norte del Istmo de Tehuantepec, y terminal sur en el Núcleo de Centroamérica; G = Terminal norte de extensión en el Núcleo de Centroamérica, y terminal sur en Sur América; $\mathrm{H}$ = Terminal norte de extensión en el Núcleo de Centroamérica, y terminal sur en Centro América, al sur de la Depresión Nicaragüense; I = Limitado al Núcleo de Centroamérica (excluyendo las endémicas hondureñas); $\mathrm{J}=$ Endémica de Honduras; $\mathrm{M}=$ Especie introducida.

\begin{tabular}{|c|c|c|c|c|c|c|c|c|c|c|c|}
\hline \multirow[t]{2}{*}{ Groups } & \multicolumn{11}{|c|}{ Broad Patterns of Distribution } \\
\hline & A & $\mathrm{B}$ & $\mathrm{C}$ & $\mathrm{D}$ & $\mathrm{E}$ & $\mathrm{F}$ & G & $\mathrm{H}$ & I & $\mathrm{J}$ & M \\
\hline Salamanders (4) & - & - & - & - & - & 1 & - & - & 3 & - & - \\
\hline Anurans (27) & 2 & 3 & 1 & 5 & 5 & - & - & 2 & 6 & 3 & - \\
\hline Turtles (4) & - & - & - & 3 & 1 & - & - & - & - & - & - \\
\hline Lizards (29) & - & 1 & - & 4 & 6 & 5 & 1 & 5 & 4 & 2 & 1 \\
\hline Snakes (41) & 4 & 2 & 1 & 12 & 6 & 2 & 2 & 6 & 5 & 1 & - \\
\hline Totals (105) & 6 & 6 & 2 & 24 & 18 & 8 & 3 & 13 & 18 & 6 & 1 \\
\hline
\end{tabular}

sea level to $2600 \mathrm{~m}$. The mean elevational range for this group of 22 species is $1012.5 \mathrm{~m}$.

3. Broad distribution patterns. The data on broad distribution patterns in Table 1 are summarized in Table 2. These data indicate that the greatest number of species $(24$ or $22.9 \%$ of the total of 105 species) belong to the D category, containing those species occurring from somewhere in Mexico north of the Isthmus of Tehuantepec to somewhere in South America. The next largest categories are E, comprising those species whose ranges extend from somewhere in Mexico north of the Isthmus of Tehuantepec to somewhere in Central America south of the Nicaraguan Depression, and I, including those species restricted to Nuclear Middle America (exclusive of Honduran endemics), each with 18 species (17.1\%). Together, these three categories contain 60 species or $57.1 \%$ of the total. The remaining eight categories contain from 1 to 13 species and possess a combined number of 45 species or $42.9 \%$ of the total. Compared to both the cloud forest (Wilson \& McCranie 2004a) and rainforest habitats (Wilson \& Townsend 2006), the upland pine-oak forests lack the distinctiveness of the other two habitat types, given the relatively few endemic species ( 6 or $5.7 \%$ of total) distributed there. Nonetheless, these forests are subject to widespread environmental damage, largely through annual burning and logging. It is also the case that little attention has been paid to these forests in designing biotic reserves in Honduras. As a consequence, these forests deserve more attention from conservation biologists (see discussion below).

4. Primary microhabitat distribution. With respect to vertical positioning within the primary microhabitats, 28 species $(26.7 \%)$ were usually encountered only in arboreal situations, 67 species $(63.8 \%)$ only in terrestrial situations, and $10(9.5 \%)$ in both. In terms of occurrence in the three major microhabitats (forest proper, streamside, pondside), 68 species $(64.8 \%)$ were found only in the forest proper, eight (7.6\%) exclusively along streams, 17 (16.2\%) only around ponds, three $(2.9 \%)$ in the forest and along streams, one $(1.0 \%)$ around ponds and along streams, seven (6.7\%) in the forest and around ponds, and one (1.0\%) in the forest, around ponds, and along streams (Table 1).

When the two sets of categories, vertical positioning in primary habitat and microhabitats are combined, 13 categories result. The largest number of species (43 or $41.0 \%$ ) consists of terrestrial forest inhabitants. The next largest group ( 19 species or $18.1 \%$ ) contains the arboreal forest inhabitants, followed by the terrestrial pondside species (12 or 11.4\%) and the terrestrial forest pondside species (seven or $6.7 \%$ ). Together, these four groups contain 81 species or $77.1 \%$ of the 105 species. The remaining nine groups contain a total of 24 species or $22.9 \%$ of the total. The terrestrial forest inhabitants include one salamander, one turtle, ten lizards, and 31 snakes. The arboreal forest inhabitants include three salamanders, 12 lizards, and four snakes. The terrestrial pondside species include 12 anurans. The terrestrial forest pondside species include two anurans, two turtles, and three snakes.

5. Relative abundance. Sixty-three species $(60.0 \%)$ are categorized as being common (two salamanders, 23 anurans, one turtle, 20 lizards, and 17 snakes), 33 (31.4\%) as being infrequent (one salamander, three anurans, three turtles, five lizards, and 21 snakes), and nine $(8.6 \%)$ as being rare (one salamander, one anuran, four lizards, and three snakes).

6. Patterns of distribution among ecophysiographic areas. Wilson et al. (2001) identified four ecophysiographic areas (areas recognized from combining forest formations and physiographic regions) that contain upland pine-oak forest vegetation, the Northwestern Uplands (NWU), Southwestern Uplands (SWU), Northeastern Uplands (NEU), and Southeastern Uplands (SEU). These areas are illustrated in Figure 1. The distribution of the members of the Honduran pine-oak herpetofauna among these four ecophysiographic areas is detailed in Table 3. Examination of the data in this table allows for several conclusions, as follows:

A. The numbers of species in these four areas range from 39 (NEU) to 70 (SEU);

B. More taxa are recorded from the Southern Cordillera areas 
(SWU and SEU-89 or $84.8 \%$ of total) than those from the Northern Cordillera areas (NWU and NEU-59 or 56.2\%), the reverse of the pattern seen in cloud forest areas (Wilson $\&$ McCranie, 2003). Forty-three species $(41.0 \%)$ are found in both cordilleras;

C. The pattern of the presence of more species in the Southern Cordillera than in the Northern Cordillera is also seen in each of the major herpetofaunal groupings. Three of the four salamander species are distributed in the Southern Cordillera, whereas only two are seen in the Northern Cordillera. Only a single salamander species (Bolitoglossa mexicana) is found in both cordilleras. Of 27 anuran species, 26 occur in the Southern Cordillera, with 16 in the Northern Cordillera. Fifteen anuran species are found in both cordilleras. The pattern for the turtles is the same as that for the salamanders, with three species occurring in the Southern Cordillera and two in the Northern Cordillera; only a single species (Rhinoclemmys pulcherrima) is found in both cordilleras. For lizards, 23 spe- cies are distributed in the Southern Cordillera and 19 in the Northern Cordillera; 13 species are found in both areas. Thirtythree species of snakes are found in the Southern Cordillera and 19 in the Northern Cordillera; 13 species occur in both areas; and

D. The majority of the 105 species ( 73 or $69.5 \%$ ) are distributed in only one or two upland pine-oak forest ecophysiographic areas. On the other hand, 20 species (19.0\%) occupy all four areas. The average area occurrence is 2.0.

The number of species shared between areas ranges from 25 to 38 . In general, as the size of the herpetofaunas of the areas increases, so does the average of the herpetofaunal sizes of the areas compared to them. Thus, arranging the four areas based on the size of their respective herpetofaunas indicates the following relationship: NEU—39 species_-mean species number of compared areas, 28.0; NWU-46-27.6; SWU-57-29.2; SEU-70-34.3. Also in general, the greater the total herpetofauna of any two compared areas, the higher the number of species shared.

Table 3. Distribution of the Honduran upland pine-oak forest herpetofauna within four ecophysiographic areas. Abbreviations are: $\mathrm{W}=\mathrm{widespread}$ in that area; $\mathrm{R}=$ restricted to that area; $\mathrm{P}=$ peripherally distributed in that area; NWU = Northwestern Uplands; SWU = Southwestern Uplands; YU = Yojoa Uplands; $\mathrm{NEU}=$ Northeastern Uplands; SEU = Southeastern Uplands.

Tabla 3. Distribución de la herpetofauna hondureña de bosques de pino-roble del altiplano dentro de cuatro áreas ecofisiográficas. Las abreviaturas son, de aduerdo a sus siglas en inglés: $\mathrm{W}=$ distribución extensa en esa área; $\mathrm{R}=$ limitado a esa área; $\mathrm{P}=$ distribución periférica en esa área; $\mathrm{NWU}=\mathrm{Altiplano}$ del Noroeste; SWU = Altiplano del Suroeste; YU = Altiplano de Yojoa; NEU =Altiplano del Nordeste; SEU = Altiplano del Sureste.

\begin{tabular}{|c|c|c|c|c|c|}
\hline Species & NWU & SWU & NEU & SEU & Totals \\
\hline \multicolumn{6}{|c|}{ Salamanders (4 species) } \\
\hline Bolitoglossa mexicana & $\mathrm{X}$ & $\mathrm{X}$ & $\mathrm{X}$ & $\mathrm{X}$ & 4 \\
\hline Bolitoglossa occidentalis & - & $X$ & - & - & 1 \\
\hline Bolitoglossa rufescens & $\mathrm{X}$ & - & - & - & 1 \\
\hline Oedipina ignea & - & $\mathrm{X}$ & - & - & 1 \\
\hline \multicolumn{6}{|c|}{ Anurans (27 species) } \\
\hline Chaunus marinus & $\mathrm{X}$ & $\mathrm{X}$ & - & $X$ & 3 \\
\hline Ollotis coccifer & - & - & $\mathrm{X}$ & $\mathrm{X}$ & 2 \\
\hline Ollotis ibarrai & - & $X$ & - & - & 1 \\
\hline Ollotis luetkenii & - & $\mathrm{X}$ & - & $\mathrm{X}$ & 2 \\
\hline Ollotis valliceps & $\mathrm{X}$ & $X$ & $\mathrm{X}$ & $\mathrm{X}$ & 4 \\
\hline Hyalinobatrachium fleischmanni & - & $\mathrm{X}$ & $X$ & $\mathrm{X}$ & 3 \\
\hline Dendropsophus microcephalus & $X$ & - & $\mathrm{X}$ & $X$ & 3 \\
\hline Plectrohyla guatemalensis & - & $X$ & - & - & 1 \\
\hline Ptychohyla hypomykter & - & $\mathrm{X}$ & - & $\mathrm{X}$ & 2 \\
\hline Ptychohyla salvadorensis & - & $\mathrm{X}$ & - & $\mathrm{X}$ & 2 \\
\hline Scinax staufferi & $\mathrm{X}$ & $\mathrm{X}$ & X & $\mathrm{X}$ & 4 \\
\hline Smilisca baudinii & $X$ & $X$ & $\mathrm{X}$ & $\mathrm{X}$ & 4 \\
\hline Tlalocohyla loquax & $\mathrm{X}$ & - & $X$ & $\mathrm{X}$ & 3 \\
\hline Trachycephalus venulosus & $\mathrm{X}$ & $\mathrm{X}$ & $\mathrm{X}$ & $X$ & 4 \\
\hline Craugastor anciano & - & $\mathrm{X}$ & - & - & 1 \\
\hline Craugastor emleni & - & - & - & $\mathrm{X}$ & 1 \\
\hline Craugastor laevissimus & - & $X$ & - & $X$ & 2 \\
\hline Leptodactylus fragilis & $\mathrm{X}$ & $X$ & $\mathrm{X}$ & $\mathrm{X}$ & 4 \\
\hline Leptodactylus melanonotus & $\mathrm{X}$ & $\mathrm{X}$ & - & $X$ & 3 \\
\hline Leptodactylus silvanimbus & - & $X$ & - & - & 1 \\
\hline Engystomops pustulosus & $X$ & $X$ & $X$ & $\mathrm{X}$ & 4 \\
\hline Hypopachus barberi & - & $X$ & - & - & 1 \\
\hline Hypopachus variolosus & $\mathrm{X}$ & $\mathrm{X}$ & $\mathrm{X}$ & $\mathrm{X}$ & 4 \\
\hline Lithobates brownorum & $\mathrm{X}$ & $\mathrm{X}$ & $\mathrm{X}$ & $X$ & 4 \\
\hline
\end{tabular}


Table 3. Continued....

\begin{tabular}{|c|c|c|c|c|c|}
\hline Species & NWU & SWU & NEU & SEU & Totals \\
\hline Lithobates forreri & - & $\mathrm{X}$ & - & - & 1 \\
\hline Lithobates maculatus & $\mathrm{X}$ & $\mathrm{X}$ & $\mathrm{X}$ & $\mathrm{X}$ & 4 \\
\hline Lithobates vaillanti & $\mathrm{X}$ & - & $\mathrm{X}$ & - & 2 \\
\hline \multicolumn{6}{|c|}{ Turtles (4 species) } \\
\hline Trachemys venusta & - & - & $\mathrm{X}$ & - & 1 \\
\hline Rhinoclemmys pulcherrima & - & - & $\mathrm{X}$ & $\mathrm{X}$ & 2 \\
\hline Kinosternon leucostomum & $\mathrm{X}$ & - & - & - & 1 \\
\hline Kinosternon scorpioides & - & $\mathrm{X}$ & - & $\mathrm{X}$ & 2 \\
\hline \multicolumn{6}{|c|}{ Lizards (29 species) } \\
\hline Celestus bivittatus & - & $\mathrm{X}$ & - & - & 1 \\
\hline Celestus scansorius & - & - & $\mathrm{X}$ & - & 1 \\
\hline Hemidactylus frenatus & - & - & - & $\mathrm{X}$ & 1 \\
\hline Phyllodactylus tuberculosus & - & - & $\mathrm{X}$ & $\mathrm{X}$ & 2 \\
\hline Sphaerodactylus glaucus & $\mathrm{X}$ & - & - & - & 1 \\
\hline Sphaerodactylus millepunctatus & $\mathrm{X}$ & - & $\mathrm{X}$ & - & 2 \\
\hline Basiliscus vittatus & $\mathrm{X}$ & $\mathrm{X}$ & $\mathrm{X}$ & $\mathrm{X}$ & 4 \\
\hline Corytophanes percarinatus & - & $\mathrm{X}$ & - & - & 1 \\
\hline Laemanctus longipes & $\mathrm{X}$ & - & - & - & 1 \\
\hline Ctenosaura similis & - & $\mathrm{X}$ & $\mathrm{X}$ & $\mathrm{X}$ & 3 \\
\hline Iguana iguana & $\mathrm{X}$ & - & - & - & 1 \\
\hline Sceloporus malachiticus & - & $\mathrm{X}$ & - & $\mathrm{X}$ & 2 \\
\hline Sceloporus olloporus & $\mathrm{X}$ & $\mathrm{X}$ & $\mathrm{X}$ & $\mathrm{X}$ & 4 \\
\hline Sceloporus squamosus & $\mathrm{X}$ & $\mathrm{X}$ & - & $\mathrm{X}$ & 3 \\
\hline Anolis biporcatus & $\mathrm{X}$ & - & - & $\mathrm{X}$ & 2 \\
\hline Anolis crassulus & - & $\mathrm{X}$ & - & - & 1 \\
\hline Anolis dariense & - & - & $\mathrm{X}$ & $\mathrm{X}$ & 2 \\
\hline Anolis laeviventris & - & $\mathrm{X}$ & - & - & 1 \\
\hline Anolis rodriguezii & $\mathrm{X}$ & - & - & - & 1 \\
\hline Anolis sericeus & $\mathrm{X}$ & $\mathrm{X}$ & $\mathrm{X}$ & $\mathrm{X}$ & 4 \\
\hline Anolis sminthus & - & - & - & $\mathrm{X}$ & 1 \\
\hline Anolis tropidonotus & $\mathrm{X}$ & $\mathrm{X}$ & $\mathrm{X}$ & $\mathrm{X}$ & 4 \\
\hline Mabuya unimarginata & $\mathrm{X}$ & $\mathrm{X}$ & $\mathrm{X}$ & $\mathrm{X}$ & 4 \\
\hline Mesoscincus managuae & - & - & - & $\mathrm{X}$ & 1 \\
\hline Sphenomorphus cherriei & $\mathrm{X}$ & - & - & $\mathrm{X}$ & 2 \\
\hline Gymnophthalmus speciosus & - & - & - & $\mathrm{X}$ & 1 \\
\hline Ameiva undulata & $\mathrm{X}$ & $\mathrm{X}$ & $\mathrm{X}$ & $\mathrm{X}$ & 4 \\
\hline Aspidoscelis deppii & - & - & - & $\mathrm{X}$ & 1 \\
\hline Aspidoscelis motaguae & $\mathrm{X}$ & - & - & $\mathrm{X}$ & 2 \\
\hline \multicolumn{6}{|c|}{ Snakes (41 species) } \\
\hline Leptotyphlops phenops & $\mathrm{X}$ & $\mathrm{X}$ & - & - & 2 \\
\hline Typhlops costaricensis & - & - & - & $\mathrm{X}$ & 1 \\
\hline Boa constrictor & - & $\mathrm{X}$ & - & - & 1 \\
\hline Ungaliophis continentalis & - & - & - & $\mathrm{X}$ & 1 \\
\hline Adelphicos quadrivirgatum & - & $\mathrm{X}$ & $\mathrm{X}$ & - & 2 \\
\hline Coniophanes fissidens & $X$ & - & - & - & 1 \\
\hline Coniophanes piceivittis & $X$ & - & - & $\mathrm{X}$ & 2 \\
\hline Conophis concolor & - & $\mathrm{X}$ & $\mathrm{X}$ & $\mathrm{X}$ & 3 \\
\hline Dendrophidion percarinatum & - & - & $\mathrm{X}$ & - & 1 \\
\hline Drymarchon melanurus & $X$ & $\mathrm{X}$ & $\mathrm{X}$ & $\mathrm{X}$ & 4 \\
\hline Drymobius margaritiferus & $\mathrm{X}$ & - & $\mathrm{X}$ & $\mathrm{X}$ & 3 \\
\hline
\end{tabular}


Table 3. Continued....

\begin{tabular}{|c|c|c|c|c|c|}
\hline Species & NWU & SWU & NEU & SEU & Totals \\
\hline Enulius flavitorques & - & - & - & $X$ & 1 \\
\hline Imantodes cenchoa & $\mathrm{X}$ & - & - & - & 1 \\
\hline Lampropeltis triangulum & $\mathrm{X}$ & - & - & - & 1 \\
\hline Leptodeira annulata & $\mathrm{X}$ & $\mathrm{X}$ & $\mathrm{X}$ & $\mathrm{X}$ & 4 \\
\hline Leptodrymus pulcherrimus & - & - & - & $\mathrm{X}$ & 1 \\
\hline Leptophis mexicanus & $\mathrm{X}$ & $\mathrm{X}$ & - & $\mathrm{X}$ & 3 \\
\hline Masticophis mentovarius & - & - & $\mathrm{X}$ & $\mathrm{X}$ & 2 \\
\hline Mastigodryas dorsalis & - & $\mathrm{X}$ & - & $\mathrm{X}$ & 2 \\
\hline Ninia sebae & $\mathrm{X}$ & $\mathrm{X}$ & $\mathrm{X}$ & $\mathrm{X}$ & 4 \\
\hline Oxybelis aeneus & $\mathrm{X}$ & - & - & - & 1 \\
\hline Pliocercus elapoides & $\mathrm{X}$ & - & - & - & 1 \\
\hline Rhadinaea godmani & - & - & - & $\mathrm{X}$ & 1 \\
\hline Rhadinaea kinkelini & - & $\mathrm{X}$ & - & - & 1 \\
\hline Scolecophis atrocinctus & - & $\mathrm{X}$ & - & - & 1 \\
\hline Senticolis triaspis & - & $\mathrm{X}$ & - & $\mathrm{X}$ & 2 \\
\hline Sibon dimidiatus & - & - & - & $\mathrm{X}$ & 1 \\
\hline Spilotes pullatus & $\mathrm{X}$ & - & $\mathrm{X}$ & $\mathrm{X}$ & 3 \\
\hline Stenorrhina degenhardtii & - & - & - & $\mathrm{X}$ & 1 \\
\hline Stenorrhina freminvillii & $\mathrm{X}$ & $\mathrm{X}$ & $\mathrm{X}$ & $\mathrm{X}$ & 4 \\
\hline Storeria dekayi & - & $\mathrm{X}$ & $\mathrm{X}$ & $\mathrm{X}$ & 3 \\
\hline Tantilla armillata & - & - & - & $\mathrm{X}$ & 1 \\
\hline Tantilla lempira & - & - & - & $\mathrm{X}$ & 1 \\
\hline Tantilla taeniata & - & - & - & $X$ & 1 \\
\hline Thamnophis fulvus & - & $X$ & - & - & 1 \\
\hline Thamnophis proximus & - & - & - & $\mathrm{X}$ & 1 \\
\hline Tretanorhinus nigroluteus & - & - & - & $\mathrm{X}$ & 1 \\
\hline Tropidodipsas fischeri & - & $\mathrm{X}$ & - & - & 1 \\
\hline Micrurus nigrocinctus & - & $\mathrm{X}$ & - & $\mathrm{X}$ & 2 \\
\hline Crotalus durissus & - & $\mathrm{X}$ & - & $\mathrm{X}$ & 2 \\
\hline Porthidium ophryomegas & - & - & - & $\mathrm{X}$ & 1 \\
\hline Total (105 species) & 46 & 57 & 39 & 70 & - \\
\hline
\end{tabular}

Calculation of Coefficient of Biogeographic Resemblance (CBR) values promotes a more robust analysis of herpetofaunal relationships. A matrix of CBR values for the four ecophysiographic areas is presented in Table 4. These values are used to produce a CBR diagram (Figure 2) indicating how the highest CBR values connect the four areas to one another. This diagram indicates that almost the same CBR value ( 0.60 or 0.61 ) connects these areas to one another, although the shared species involved are different in each case. It also demonstrates that the two Northern Cordillera areas are closely related to one another, as are the two Southern Cordillera areas. The Northern Cordillera and the Southern Cordillera areas are connected to one another through the NEU and SEU areas. The NWU and SWU areas are connected to one another by a decidedly lower CBR value of 0.49 , the lowest value for either area (Table 4). Finally, even though the NWU and SEU areas share a higher number of species (32) than do the NWU and NEU areas (26), the CBR value is lower for the former (0.55) than for the latter (0.61).

Averaging CBR values for each area gives a measure of herpetofaunal distinctiveness, as follows: NWU-0.55; SWU-0.54; NEU- 0.58 ; SEU -0.59 . These figures are relatively close to one another, compared to those for the cloud forest areas reported by Wilson
\& McCranie (2004a), and are indicative of the relative homogeneity of the herpetofaunas of these four upland pine-oak areas and the very low level of endemicity within these areas. As noted above, only six of the 105 species are endemic to the country, each of these occupying only one upland pine-oak forest ecophysiographic area.

\section{Biodiversity significance and conservation status of the upland pine-oak forest herpetofauna}

The herpetofauna of the upland pine-oak forests of Honduras is subject to the same anthropogenic pressures as those outlined for the nation's herpetofauna by Wilson \& McCranie (2004b), as well as for its cloud forest (Wilson \& McCranie 2004a) and rainforest (Wilson \& Townsend 2006) herpetofaunas. The areas supporting upland pine-oak forests were well populated in pre-Columbian times and were the first (and for a prolonged period the only) parts of Honduras settled by the Spanish (Tompson 2001). For this reason, it can be difficult to fully gauge the impact humans have had on the biodiversity of the upland pine-oak forests, or whether the modern forests even resemble the forests that were present prior to modern disturbance. There can be little doubt that the seasonal burning of large areas of 
Table 4. CBR matrix of herpetofaunal relationships for the four ecophysiographic areas supporting upland pine-oak forest. $\mathbf{N}=$ species in each region; $\mathrm{N}=$ species in common between two regions; $\underline{\mathrm{N}}=$ Coefficients of Biogeographic Resemblance. See text for explanation of the abbreviations.

Tabla 4. Tabla 4. Matriz de CBR de las relaciones entre la herpetofauna de las cuatro áreas ecofisiográficas que contienen bosques de pino-roble de altiplano. $\mathrm{N}$ = especies en cada región; $\mathrm{N}=$ especies en común entre dos regiones; $\mathrm{N}=$ Coeficientes de Semejanza Biogeográfica (CBR). Ver texto para explanaciones de las abreviaturas.

\begin{tabular}{|c|c|c|c|c|}
\hline & NWU & SWU & NEU & SEU \\
\hline NWU & 46 & 25 & 26 & 32 \\
\hline SWU & $\underline{0.49}$ & 57 & 25 & 38 \\
\hline NEU & $\underline{0.61}$ & $\underline{0.52}$ & 39 & 33 \\
\hline SEU & $\underline{0.55}$ & $\underline{0.60}$ & $\underline{0.61}$ & 70 \\
\hline
\end{tabular}

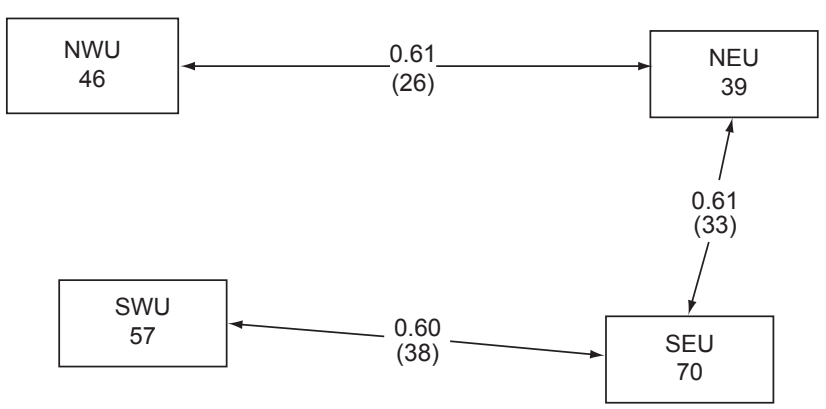

Figure 2. Coefficient of Biogeographic Resemblance for the four upland pine-oak forest areas in Honduras. Abbreviations are as follows: NWU = Northwestern Uplands; NEU = Northeastern Uplands; SWU = Southwestern Uplands; SEU = Southeastern Uplands. Numbers in boxes are the number of species in each area; decimal numbers on arrows indicate the CBR value shared between areas connected and represent the highest value for each area; absolute numbers in parentheses indicate the number of species shared between the areas connected. Position of the boxes in the diagram is roughly reflective of their geographic relationships in Honduras.

Figura 2. Coeficiente de Semblanza Biogeográfica (CBR) de las cuatro áreas de bosques de pino-roble del altiplano en Honduras. Las abreviaturas son las siguientes, de acuerdo sus siglas en inglés: NWU = Altiplano del Noroeste; NEU =Altiplano del Nordeste; SWU = Altiplano del Suroeste; SEU = Altiplano del Sureste. Los números en los cuadros son el número de especies en cada área; los números decimales sobre las flechas indican el valor de CBR compartido entre las áreas conectadas y representan el valor más alto de cada área; los números absolutos en paréntesis indican el número de especies compartidas entre las áreas conectadas. La posición de los cuadros en el diagrama refleja aproximadamente sus relaciones geográficas en Honduras.

the upland pine-oak forest and widespread logging and land clearing have definitely shaped the constituency of the extant upland pine-oak forest herpetofauna. A further, albeit natural, cause of disturbance in the upland pine-oak forests has been pine bark beetle infestation. In the early 1960's, an epidemic infestation of the pine bark beetle Dendroctonus frontalis swept through the Honduran pine-oak forests, causing tree mortality at around $25 \%$ nationwide and as high as $70 \%$ in localized areas (Coyne \& Critchfield 1974).

Both Wilson \& McCranie (2004a) and Wilson \& Townsend (2006) considered the species with the greatest biodiversity significance to be those that are endemic to Honduras or otherwise restricted to Nuclear Middle America. As mentioned previously, the upland pine-oak forests lack the high numbers of endemic species that are seen in the rainforests and cloud forests. As indicated in Table 2, six species known from these forests are endemic to the country, a figure that is just $5.7 \%$ of the total of 105 . An additional 18 species (17.1\%) are otherwise restricted to Nuclear Middle America. Together, these 24 species make up $22.9 \%$ of the upland pine-oak forest herpetofauna, comparable in ratio to the much more speciose rainforest herpetofauna ( 65 species or $26.2 \%$ of the total), but nowhere near the diversity demonstrated by the cloud forest herpetofauna ( 83 species or $68.0 \%$ of the total).

We have used the same standard categories employed by Wilson \& McCranie (2004a) and Wilson \& Townsend (2006, In press) in our analysis of the conservation status of the Honduran upland pine-oak forest herpetofauna (see Table 1). Sixty-two species (59.0\%) are reported to have stable populations in at least one upland pine-oak forest locality (three salamanders, 20 anurans, two turtles, 20 lizards, and 17 snakes). The fact that such a relatively high percentage of the upland pine-oak forest herpetofauna exhibits stable populations may indicate that this habitat is primarily populated by species that are able to survive in drier and less hospitable environments and, therefore, may be less susceptible to the effects deforestation.

Only four species $(3.8 \%)$ have all known Honduran populations in decline (the anurans Plectrohyla guatemalensis and Leptodactylus silvanimbus, and the snakes Crotalus simus and Porthidium ophryomegas). The two snake species, both venomous and easily recognizable, are declining due to human persecution.

Two species $(1.9 \%)$, the anurans Craugastor anciano and C. emleni, are considered extinct (McCranie \& Wilson 2002; Wilson \& McCranie 2004a and b), as neither of these species has been seen in over 15 years, despite repeated efforts to locate them at localities where they were previously found. The reason for their disappearance is not clear; all that is clear is that most populations of streamside Craugastor from above $900 \mathrm{~m}$ elevation in Honduras have either suffered noticeable declines or disappeared altogether (McCranie \& Wilson 2002; Wilson \& McCranie 2004a and b).

Thirty-five species $(33.3 \%)$ lack sufficient data for an accurate estimate of their conservation status to be made (one salamander, four anurans, two turtles, nine lizards, and 22 snakes). With more than one-third of the herpetofauna of the pine-oak forests placed in the category "no data on population status available," it is apparent that considerable fieldwork is still needed before the conservation issues relating to this group of amphibians and reptiles can be properly addressed.

\section{Acknowledgments}

The first author has been involved in fieldwork and research in Honduras since 1967 and the second author, once a student of the first author, since 1999. During this time, we have incurred indebtedness to a large number of individuals. James R. McCranie was the perennial coworker and companion while undertaking fieldwork in Honduras, and some of the data included in this paper was included in an unpublished manuscript coauthored by McCranie and LDW. We were also assisted by Kenneth L. Williams, John R. Meyer, Mario R. Espinal, Steve W. Gotte, Louis Porras, Gordon W. Schuett, Donald E. Hahn, and a number of other individuals. During the preparation of this paper, JHT was supported by a Tropical Conservation and Development Graduate Fellowship and by the 
Reptile and Amphibian Conservation Corps and the Herpetology Division of the Florida Museum of Natural History. We were furnished collecting and export permits over the years by numerous personnel of the now-defunct Recursos Naturales Renovables and its subsequent replacement, Corporación Hondureña de Desarrollo Forestal (COHDEFOR).

\section{References}

AGÜDELO, C.N. 1987. Ecosistemas terrestres de Honduras. Asociación Hondureña de Ecología, Tegucigalpa, Honduras.

CAMPBELL, J.A. 1999. Distribution patterns of amphibians in Middle America. In Patterns of distribution of amphibians. A global perspective (ed. W. E. Duellman, ed.), John Hopkins University Press, Baltimore, Maryland, p. 111-210.

CAMPBELL, J.A. \& LAMAR, W.W. 2004. The venomous reptiles of the Western Hemisphere. Comstock Publishing Associates, Ithaca, New York. Vol. I: i-xviii, 1-476 [1-28], plates 1-751; Vol. II: i-xiv, 477-616 [1-27], plates 752-1365.

COYNE, J.F. \& CRITCHFIELD, W.B. 1974. Identity and terpene composition of Honduras pines attacked by the bark beetle Dendroctonus frontalis (Scolytidae). Turrialba 24:327-331.

CRAWFORD, A.J. \& SMITH, E.N. 2005. Cenozoic biogeography and evolution in direct-developing frogs of Central America (Leptodactylidae: Eleutherodactylus) as inferred from a phylogenetic analysis of nuclear and mitochondrial genes. Molecular Phylogenetics and Evolution 35:536-555.

DIXON, J.R. \& TIPTON, B.L. 2004. Dryadophis versus Mastigodryas (Ophidia: Colubridae): a proposed solution. Herpetological Review 35: 347-349.

DUELLMAN, W.E. 1990. Herpetofaunas in neotropical rainforests: comparative composition, history, and resource use. In Four neotropical rainforests (A. H Gentry, ed.), Yale University Press, New Haven, Connecticut, p. $455-505$.

FAIVOVICH, J.C., F.B. HADDAD, P.C.A., GARCIA, D.R., FROST, J.A., CAMPBELl, \& Wheeler, W.C. 2005. Systematic review of the frog family Hylidae, with special reference to Hylinae: phylogenetic analysis and taxonomic revision. Bulletin American Museum of Natural History 294:1-240.

FARJON, A.J.A., PEREZ DE LA ROSA, \& B.T. Styles. 1997. A field guide to the pines of Mexico and Central America. England Royal Botanical Gardens, Kew.

FROST, D.R.T., GRANT, J., FAIVOVICH, R.H., Bain, A., HAAS, C.F.B., HADDAD, R.O., DE SÁ, A., CHANING, M., WILKINSON, S.C., DONNELLAN, C. J., RAXWORTHY, J.A., CAMPBELL, B.L., BLOTO, P., MOLER, R.C., DREWES, R.A., NUSSBAUM, J.D., LYNCH, D.M. GREEN \& WHEELER, W.C. 2006a. The amphibian tree of life. Bulletin American Museum of Natural History 297:1-370.

FROST, D.R.T. GRANT, \& J.R. MENDELSON, III. 2006b. Ollotis Cope, 1875 is the oldest name for the genus currently referred to as Cranopsis Cope, 1875 (Anura: Hyloides: Bufonidae). Copeia 2006(3):558.

GUYER, C. \& SAVAGE, J.M. 1986. Cladistic relationships among anoles (Sauria: Iguanidae). Systematic Zoology 35:509-531.

GUYER, C. \& SAVAGE, J.M. 1992. Anole systematics revisited. Systematic Biology 41:89-110.

HOLDRIDGE, L. R. 1967. Life zone ecology. Revised ed. Tropical Science Center, San José, Costa Rica.
MCCRANIE, J.R., TOWNSEND, J.H. \& Wilson. L.D. 2006. The amphibians and reptiles of the Honduran Mosquitia. Krieger Publishing, Melbourne, Florida.

MCCRANIE, J.R. \& WILSON, L.D. 2002. The amphibians of Honduras. Society Study of Amphibians and Reptiles, Contributions in Herpetology.

MEYER, J.R. \& Wilson, L.D. 1971. A distributional checklist of the amphibians of Honduras. Los Angeles County Museum of Natural History, Contributions in Science 218:1-47.

MEYER, J.R. \& L.D. WILSON. 1973. A distributional checklist of the turtles, crocodilians, and lizards of Honduras. Los Angeles County Museum of Natural History, Contributions in Science 244:1-39.

POE, S. 2004. Phylogeny of anoles. Herpetological Monographs 18: 37-89.

TOMPSON, D.A. 2001. Frontiers of identity: The Atlantic Coast and the formation of Honduras and Nicaragua, 1786-1894. Ph.D. Thesis. University of Florida, Gainesville.

WAGNER, P.L. 1964. Natural vegetation of Middle America. Pp. 216-264. In West, R.C. Natural environment and early cultures. Volume 1. In Wauchope, R. Handbook of Middle American Indians. University of Texas Press, Austin. 570 p.

WILSON, L.D. \& McCRANIE, J.R. 2004a. The herpetofauna of the cloud forests of Honduras. Amphibian and Reptile Conservation 3: 34-48.

WILSON, L.D. \& McCRANIE, J.R. 2004b. The conservation status of the herpetofauna of Honduras. Amphibian and Reptile Conservation 3:6-33.

WILSON, L.D., McCRANIE, J.R. \& ESPINAL, M.R. 2001. The ecogeography of the amphibians and reptiles of Honduras and the design of herpetofaunal reserves. In Mesoamerican herpetology: Systematics, zoogeography, and conservation, eds. J.D. Johnson, R.G. Webb, and O.A. Flores-Villela, 109-158. Centennial Museum, El Paso, Texas.

WILSON, L.D. \& MEYER, J.R. 1985. The snakes of Honduras. Second edition. Milwaukee Public Museum, Milwaukee, Wisconsin.

WILSON, L.D. \& TOWNSEND, J.H. 2006. The herpetofauna of the rainforests of Honduras. Caribbean Journal of Science 42:88-113.

WILSON, L.D. \& TOWNSEND, J.H. In press. Biogeography and conservation of the herpetofauna of the subhumid forests of Honduras. Studies on Neotropical Fauna and Environment.

ZALDÍVAR-RIVERÓN, A.V., LEÓN-REGAGNON, \& NIETO-MONTES DE OCA, A. 2004. Phylogeny of the Mexican coastal leopard frogs of the Rana berlandieri group based on mtDNA sequences. Molecular Phylogenetics and Evolution 30:38-49.

Title: Biogeography and conservation of the herpetofauna of the Upland Pine-Oak Forests of Honduras.

Authors: Wilson, LD e Townsend, JH

Biota Neotropica, Vol.7 (number 1): 2007

http://www.biotaneotropica.org.br/v7n1/pt/abstract?inventory+ bn02307012007

Data Received 09/06/06 - Revised 01/12/06 -

Accepted 20/01/07

ISSN 1676-0603 